\title{
分散的第二相粒子对 $\mathrm{LiCl}$ 电导率的影响
}

\author{
陈立泉 赵宗源王刚 李子荣 \\ (中国科学院物理研究所)
}

近几年来, 对超离子导体的研究日益增多, 然而能称为超离子导体的材料却为数很少. 材 料科学家的一个重要任务就是寻找离子电导率高的材料. 目前普遍采用的方法是合成具有所 谓敞形结构的化合物. 其它方法已开始引起人们的重视.

1973 年, Liang ${ }^{[11}$ 报道了在 $\mathrm{LiI}$ 中加入 $35-45 \mathrm{~m} / 0 r-\mathrm{Al}_{2} \mathrm{O}_{3}$ 时, 在室温下锂离子电导率比纯 $\mathrm{LiI}$ 的高两个数量级. 由于 $r-\mathrm{Al}_{2} \mathrm{O}_{3}$ 并未溶于 $\mathrm{LiI}$ 中, 也末与 $\mathrm{LiI}$ 形成新相, 因此把它称为第 二相粒子. 1979 年, Alpen ${ }^{[2]}$ 重复了 Liang 的实验, 认为加人 $r-\mathrm{Al}_{2} \mathrm{O}_{3}$ 后, 其电导率只提高了一 个数量级. 同年, $\mathrm{Jow}^{[3]}$ 测量了加人 $r-\mathrm{Al}_{2} \mathrm{O}_{3}$ 的 $\mathrm{CuCl}$ 的离子电导率, 发现室温铜离子电导率增 加呐个数量级,并且认为第二相粒子的颗粒度对电导率的提高有着重要的影响.

迄今为止, 都以 $r-\mathrm{Al}_{2} \mathrm{O}_{3}$ 作为第二相粒子. 除了研究电导性能而外, 尚未作其它性能研究. 是不是可以用其它绝缘材料作为第二相, 加人第二相以后对其它性能有无影响? 这就是本文。 要解决的问题.

我们用离子晶体 $\mathrm{LiCl}$ 作为基质材料. 采用 $\alpha-\mathrm{Al}_{2} \mathrm{O}_{3}, r-\mathrm{Al}_{2} \mathrm{O}_{3}$ 和 $\eta-\mathrm{Al}_{2} \mathrm{O}_{3}$ 以及 $\mathrm{SiO}_{2}$ 作为 第二相. 其颗粒度估计不小于 $3 \mu \mathrm{m}$. 将化学纯 $\mathrm{LiCl}$ 和上述各种第二相化合物分别在 $100-$ $120^{\circ} \mathrm{C}$ 烘干后, 分别研磨, 按所需的克分子百分比称量, 充分研磨混匀后经 $10000 \mathrm{~kg} / \mathrm{cm}^{2}$ 压成 直径 $12 \mathrm{~mm}$, 厚 $1.5-3.0 \mathrm{~mm}$ 的圆片. 上述操作都是在干燥箱中进行的. 然后将纯 $\mathrm{LiCl}$ 圆片 和加人第二相的圆片在 $\mathrm{N}_{2}$ 气流中分别在 $550^{\circ} \mathrm{C}$ 和 $620^{\circ} \mathrm{C}$ 烧结二小时, 快速冷却到室温. ‘加 人第二相的样品烧结温度虽然已超过 $\mathrm{LiCl}$ 的熔点, 但仍保持初始形状, 这可能是 $\mathrm{LiCl}$ 熔体对 第二相浸润和表面张力所致.

$\mathrm{X}$ 射线分析表明: 分散的第二相粒子均未溶解到 $\mathrm{LiCl}$.中,也无新相形成.

在 $500 \mathrm{kHz}$ 和 $100-500^{\circ} \mathrm{C}$ 温度范围内测量了样品的总电导. 所用实验装置和仪器同文 献[4].

纯 $\mathrm{LiCl}$ 和含 $25 \mathrm{~m} / 0$ 各种第二相粒子 $\mathrm{LiCl}$ 的电导率与温度的关系示于图 1 中. 表 1 中给 出了各种样品的电导率和电导激活能数据. $\mathrm{LiCl}+r-\mathrm{Al}_{2} \mathrm{O}_{3}$ 样品的电导率与 $r-\mathrm{Al}_{2} \mathrm{O}_{3}$ 含量的 关系示于图 1 的插入图中. 电导率的测量结果可以汇总如下:

1. 与纯 $\mathrm{LiCl}$ 相比, 含第二相粒子的 $\mathrm{LiCl}$ 样品的离子电导率都显著增加, 以含 $25 \mathrm{~m} / 0 \eta-$ $\mathrm{Al}_{2} \mathrm{O}_{3}$ 的 $\mathrm{LiCl}$ 样品为最明显. 在温度低于 $280^{\circ} \mathrm{C}$ 时, 含 $25 \mathrm{~m} / 0 \eta-\mathrm{Al}_{2} \mathrm{O}_{3}$ 的 $\mathrm{LiCl}$ 的离子电导率增 加了约二个数量级.

2. $\mathrm{LiCl}+r-\mathrm{Al}_{2} \mathrm{O}_{3}$ 样品的离子电导率与 $r-\mathrm{Al}_{2} \mathrm{O}_{3}$ 含量有关. 在 $25 \mathrm{~m} / 0 r-\mathrm{Al}_{2} \mathrm{O}_{3}$ 附近出现 最大值.

3. 含各种第二相粒子的 $\mathrm{LiCl}$ 样品的电导激活能都降低, 在高温区尤为明显. 含 $25 \mathrm{~m} / 0 \eta-$

本文 1980 年 8 月 13 日收到.

第 2 期

科学 通 报 
$\mathrm{Al}_{2} \mathrm{O}_{3}$ 的电导激活能不到纯 $\mathrm{LiCl}$ 的一半.

4. 直流极化实验表明: 含各种第二相粒子的 $\mathrm{LiCl}$ 样品的电子电导率小于总电导率的 $1 \%$,因而电导率的提高主要是锂离子电导率的增加.

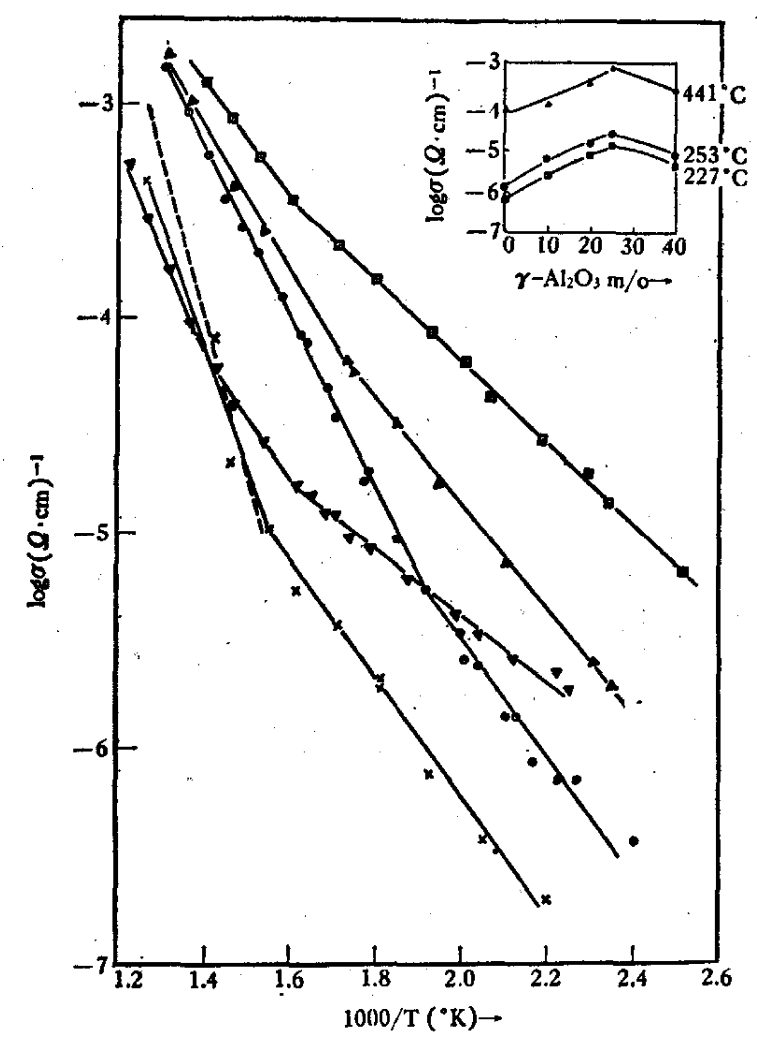

图 1 纯 $\mathrm{LiCl}$ 和含 $25 \mathrm{~m} / 0$ 第二相粒子 $\left(\alpha-\mathrm{Al}_{2} \mathrm{O}_{3}\right.$, $r-\mathrm{Al}_{2} \mathrm{O}_{3}, \eta-\mathrm{Al}_{2} \mathrm{O}_{3}$ 和 $\left.\mathrm{SiO}_{2}\right) \mathrm{LiCl}$ 的离子电导率

与温度的关系

X一一纯LiCI(本文);

- - — — 纯 $\mathrm{LiCl}$ (文献[5]);

$\mathrm{O}-\mathrm{LiCl}+25 \mathrm{~m} / 0 \mathrm{\alpha}-\mathrm{Al}_{2} \mathrm{O}_{3}$;

$\Delta-\mathrm{LiCl}+25 \mathrm{~m} / 0 \mathrm{SiO}_{2} ;$;

$\Delta-\mathrm{LiCl}+25 \mathrm{~m} / 0 \mathrm{r}-\mathrm{Al}_{2} \mathrm{O}_{3}$;

曰- $\mathrm{LiCl}+25 \mathrm{~m} / 0 \eta-\mathrm{Al}_{2} \mathrm{O}_{3}$ 。

插图: 含 $r-\mathrm{Al}_{2} \mathrm{O}_{3}$ 的 $\mathrm{LiCl}$ 的离子电导率 与 $r-\mathrm{Al}_{2} \mathrm{O}_{3}$ 含量的关系

为了解释含第二相粒子的 $\mathrm{LiCl}$ 中离子 电导率提高的机理，我们对各种样品（当时 未研究含 $\eta-\mathrm{Al}_{2} \mathrm{O}_{3}$ 的样品) 进行了 ${ }^{7} \mathrm{Li}$ 的核磁 共振线宽和自旋一晶格弛豫时间 $T_{1}$ 的研究， 其共振频率为 35 兆赫，变温范围为 -100 $-210^{\circ} \mathrm{C}$.

由核磁共振实验,我们发现:

1. 含第二相粒子的 $\mathrm{LiCl}$ 中. ${ }^{7} \mathrm{Li}$ 的核磁共 振吸收谱线上出现一附加峰. 对于 $\mathrm{LiCl}+r-$ $\mathrm{Al}_{2} \mathrm{O}_{3}$ 样品, 此小峰随 $r-\mathrm{Al}_{2} \mathrm{O}_{3}$ 的含量而变化, 以含 $75 \mathrm{~m} / 0 \mathrm{LiCl}+25 \mathrm{~m} / 0 r-\mathrm{Al}_{2} \mathrm{O}_{3}$ 的样品为 最明显 (图 2). 此小峰也随温度而变化. 对 于 $\mathrm{LiCl}+\mathrm{SiO}_{2}$ 的情况，观测到了类似的现 象. 在纯 $\mathrm{LiCl}$ 的情况中，没有观测到附加 峰.

2. 含 $r-\mathrm{Al}_{2} \mathrm{O}_{3}$ 的 $\mathrm{LiCl}$ 中 ${ }^{7} \mathrm{Li}$ 核磁共振信 号的信噪比显著提高, 而且也随 $r-\mathrm{Al}_{2} \mathrm{O}_{3}$ 的 含量而变化，也以含 $25 \mathrm{~m} / 0 r-\mathrm{Al}_{2} \mathrm{O}_{3}$ 的 $\mathrm{LiCl}$ 为最明显.

3 含 $r-\mathrm{Al}_{2} \mathrm{O}_{3}$ 的 $\mathrm{LiCl}$ 中 ${ }^{7} \mathrm{Li}$ 的自旋-晶 的格驰豫时间变短, 且随 $r-\mathrm{Al}_{2} \mathrm{O}_{3}$ 的含量而 变化. 在含第二相粒子的 LiI 中得到了类似 的结果.

这些结果表明: ${ }^{7} \mathrm{Li}$ 核磁共振吸收谱线 上附加峰的出现是由于分散的第二相粒子所 造成的. 和电导率的测量相比较, 可知小峰 表 1 纯 $\mathrm{LiCl}$ 和含第二相粒子的 $\mathrm{LiCl}$ 的离子电导率 $\sigma$ 和激活能 $E_{A}$

\begin{tabular}{l|c|c|c|c|c}
\hline \multirow{2}{*}{ 样 } & \multicolumn{3}{|c|}{ 电导率 $(\Omega \cdot \mathrm{cm})^{-1}$} & \multicolumn{2}{|c}{ 激活能 $(\mathrm{eV})$} \\
\cline { 2 - 5 } & $182^{\circ} \mathrm{C}$ & $283^{\circ} \mathrm{C}$ & $394^{\circ} \mathrm{C}$ & 低 温 区 & 高 温 区 \\
\hline \multirow{2}{*}{$\mathrm{LiCl}$} & $1.70 \times 10^{-7}$ & $2.00 \times 10^{-6}$ & $2.31 \times 10^{-3}$ & 0.42 & 1.31 \\
$\mathrm{LiCl}+25 \mathrm{~m} / 0 \mathrm{SiO}_{2}$ & $2.25 \times 10^{-6}$ & $7.50 \times 10^{-6}$ & $3.15 \times 10^{-5}$ & 0.32 & 0.83 \\
$\mathrm{LiCl}+25 \mathrm{~m} / 0 \alpha-\mathrm{Al}_{2} \mathrm{O}_{3}$ & $9.90 \times 10^{-7}$ & $1.75 \times 10^{-6}$ & $2.47 \times 10^{-4}$ & 0.39 & 0.76 \\
$\mathrm{LiCl}+25 \mathrm{~m} / 0 \mathrm{r}-\mathrm{Al}_{2} \mathrm{O}_{3}$ & $4.40 \times 10^{-6}$ & $4.10 \times 10^{-5}$ & $3.65 \times 10^{-4}$ & 0.33 & 0.63 \\
$\mathrm{LiCl}+25 \mathrm{~m} / 0 \eta-\mathrm{Al}_{2} \mathrm{O}_{3}$ & $2.53 \times 10^{-5}$ & $1.50 \times 10^{-4}$ & $6.90 \times 10^{-4}$ & 0.39 & 0.51 \\
\hline
\end{tabular}


大的其离子电导率就高. 由于 $\mathrm{LiCl}$ 含分散的第二相粒子, 自旋-晶格弛豫时间变短, 因而 ${ }^{7} \mathrm{Li}$ 核与其周围晶格场之间交换能量过程的速度加快了. 因此, 可以认为离子电导率提高的主要 原因是由于 $\mathrm{Li}^{+}$离子所处环境的改变, 这就改变了运动离子与周围环境之间的相互作用,从而 使 $\mathrm{Li}^{+}$离子扩散增强, 运动加快. 其细节将在以后报道.

附带提一下, 最近我们做了进一步的核 磁共振实验, 特别是对含 $\eta-\mathrm{Al}_{2} \mathrm{O}_{3}$ 的样品,并 得到了和电导率测量符合得更好的结果 (见 图 3).

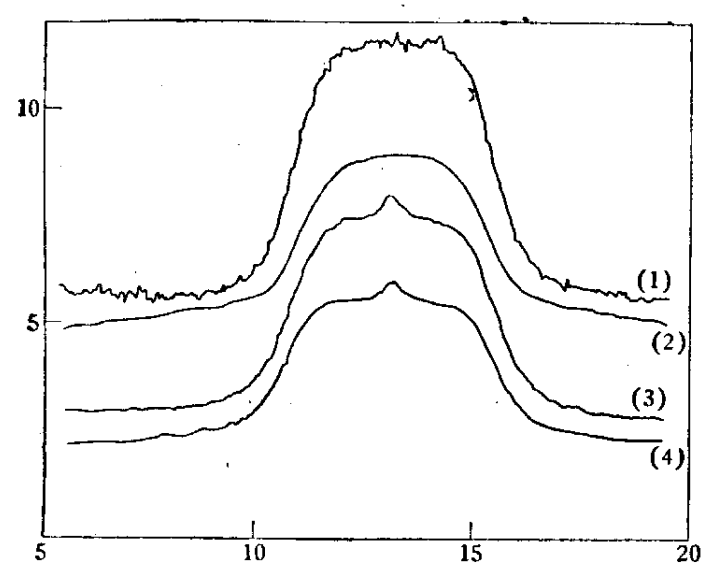

图 2 纯 $\mathrm{LiCl}$ 和 $\mathrm{LiCl}+25 \mathrm{~m} / 0 r-\mathrm{Al}_{2} \mathrm{O}_{3}$ 中 ${ }^{7} \mathrm{Li}$ 核 磁共振谱线 (频率: 35.00 兆赫, 温度: $23.5^{\circ} \mathrm{C}$ )

1. 纯 $\mathrm{LiCl}$ 累加 200 次;

2. 纯 $\mathrm{LiCl}$ 累加 671 次;

3. $\mathrm{LiCl}+25 \mathrm{~m} / 0 r-\mathrm{Al}_{2} \mathrm{O}_{3}$ 累加 200 次;

4. $\mathrm{LiCl}+25 \mathrm{~m} / 0 r-\mathrm{Al}_{2} \mathrm{O}_{3}$ 累加 271 次

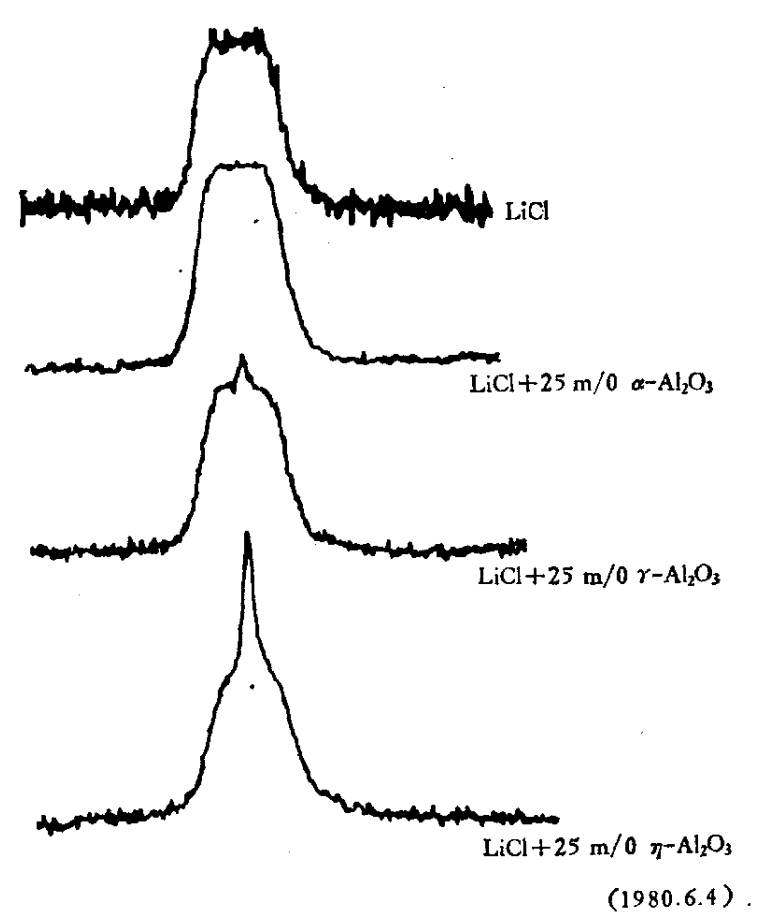

图 3 纯 $\mathrm{LiCl}, \mathrm{LiCl}+25 \mathrm{~m} / 0 \alpha-\mathrm{Al}_{2} \mathrm{O}_{3}$, $\mathrm{LiCl}+25 \mathrm{~m} / 0 \mathrm{r}-\mathrm{Al}_{2} \mathrm{O}_{3}$ 和 $\mathrm{LiCl}+25 \mathrm{~m} / 0 \eta-\mathrm{Al}_{2} \mathrm{O}_{3}$ 中 ' $\mathrm{Li}$ 核磁共振谱线 (频率: 35.00 兆赫, 温度: $27.5^{\circ} \mathrm{C}$ )

\section{参考文献}

[1] Liang, C. C., J. Electrochem. Soc., 120(1973), 1289-1292.

[ 2$\rceil$ Alpen, U. V. \& Bell, M. F., in Fast Ion Transport in Solids (eds. by Vashishta, Mundy, Shenoy), Elsevier North Holland Inc., 1979, 463-469.

[ 3 ] Jow, T. \& Bruce Wagner, Jr. J., J. Electrochem. Soc., 126(1979), 1963-1972.

[4] Chen Lichuan ete., Acta Physica Sinica, 29(1980), 661-666.

[5] Haven, Y., Rec. Trav. Chim. Pays-Bus, 69(1950), 1471. 